\title{
Co-expression network analysis of differentially expressed genes associated with metastasis in prolactin pituitary tumors
}

\author{
WEI ZHANG ${ }^{1}$, ZHENLE ZANG $^{1}$, YECHUN SONG $^{1}$, HUI YANG $^{1}$ and QING YIN $^{2}$ \\ ${ }^{1}$ Department of Neurosurgery, Xinqiao Hospital, Third Military Medical University, Chongqing 400037; \\ ${ }^{2}$ Department of Rehabilitation and Physical Therapy, Southwest Hospital, \\ Third Military Medical University, Chongqing 400038, P.R. China
}

Received August 23, 2013; Accepted March 5, 2014

DOI: $10.3892 / \mathrm{mmr} .2014 .2152$

\begin{abstract}
The aim of the present study was to construct a co-expression network of differently expressed genes (DEGs) in prolactin pituitary (PRL) tumor metastasis. The gene expression profile, GSE22812 was downloaded from the Gene Expression Omnibus database, and including five non-invasive, two invasive and six aggressive-invasive PRL tumor samples. Compared with non-invasive samples, DEGs were identified in invasive and aggressive-invasive samples using a limma package in $\mathrm{R}$ language. The expression values of DEGs were hierarchically clustered. Next, Gene Ontology (GO) function enrichment and Kyoto Encyclopedia of Genes and Genomes pathway analysis of DEGs were performed via The Database for Annotation, Visualization and Integrated Discovery. Finally, gene pairs of DEGs between non-invasive and aggressive-invasive samples were identified using the Spearman cor( ) function in $\mathrm{R}$ language. Compared with the non-invasive samples, 61 and 89 DEGs were obtained from invasive and aggressive-invasive samples, respectively. Cluster analysis showed that four genes were shared by the two samples, including upregulated solute carrier family 2 , facilitated glucose transporter member 11 (SLC2A11) and teneurin transmembrane protein 1 (TENMI) and downregulated importin 7 (IPO7) and chromogranin B $(C H G B)$. In the invasive samples, the most significant GO terms responded to cyclic adenosine monophosphate and a glucocorticoid stimulus. However, this occurred in the cell cycle, and was in response to hormone stimulation in aggressive-invasive samples. The co-expression network of DEGs showed different gene pairs and modules, and SLC2A11 and CHGB occurred in two co-expression networks within different co-expressed pairs.
\end{abstract}

Correspondence to: Dr Yechun Song or Dr Hui Yang, Department of Neurosurgery, Xinqiao Hospital, Third Military Medical University, No. 83 Xinqiaozheng Street, Shapingba, Chongqing 400037, P.R. China

E-mail: yechunsong001@hotmail.com

E-mail: huiyang001fh@hotmail.com

Key words: prolactin pituitary tumor, differentially expressed genes, tumor metastasis, co-expression network
In the present study, the co-expression network was constructed using bioinformatics methods. SLC2A11, TENM1, IPO7 and $C H G B$ are hypothesized to be closely associated with metastasis of PRL. Furthermore, $C H G B$ and SLC2A11 may be significant in PRL tumor progression and serve as molecular biomarkers for PRL tumors. However, further investigation is required to confirm the current results.

\section{Introduction}

Approximately $10 \%$ of all clinically overt intracranial neoplasms arise from the pituitary gland, of which prolactin-secreting adenomas, also termed prolactinomas, were the most common, accounting for $30-40 \%$ of pituitary tumors $(1,2)$. Prevalence has classically been indicated to be 94 per 100,000 inhabitants (3). The early diagnosis of prolactin-secreting pituitary tumors is difficult due to the early signs or symptoms being associated with the overproduction of hormones, and hormones that are only combined with a single tumor are particularly rare $(2,4)$. Hormones, such as estrogens, are apparently not the only cause and other possible hormones, including local growth factors in pituitary prolactinomas, are less well defined (5).

Prolactin pituitary (PRL) tumors are classified and managed according to size (6). For example, microprolactinomas $(<1 \mathrm{~cm})$ do not typically invade the parasellar region, while macroprolactinomas $(>1 \mathrm{~cm})$ are more likely to locally invade and compress surrounding structures. Previous studies have determined the molecular pathogenesis of pituitary tumor aggressive behavior and malignant transformation. A dopamine agonist was identified to control PRL via decreased D2 receptor availability, differential isoform expression and disrupted autocrine growth factor signaling $(7,8)$. $\operatorname{ErbB}$ receptors, epidermal growth factor receptor (9), p185her2/neu (10), ErbB3 and ErbB4 (11), including the kinase-deficient ErbB3 dimerization with p185her2/neu may reflect tumor progression to an increasingly dedifferentiated state $(12,13)$. Increased $E r b B$ receptor expression has been verified in aggressive pituitary tumors and carcinomas. Furthermore, inhibition of the $E r b B$ receptor may provide an alternative medical control of tumor growth and hormone secretion (14).

In the present study, the gene expression profile of PRL tumor samples, including non-invasive, invasive and aggressive-invasive samples, were investigated and the differentially 
expressed genes (DEGs) associated with tumor metastasis were identified. The DEGs were subsequently clustered and The Database for Annotation, Visualization and Integrated Discovery (DAVID) was used to identify over-represented Gene Ontology (GO) categories in biological processes and significant pathways. Finally, gene pairs of DEGs between non-invasive and aggressive-invasive samples were identified and used to construct the co-expression network. The present study aimed to identify co-expressed gene pairs for PRL tumor metastasis via bioinformatics methods, with the purpose of investigating potential molecular biomarkers for use in clinical treatment.

\section{Materials and methods}

Microarray data and preprocessing. The gene expression profile GSE22812 (15) was downloaded from the Gene Expression Omnibus database (http://www.ncbi.nlm.nih.gov/geo/), and included 13 PRL tumor samples. These samples were classified as non-invasive $(n=5)$, invasive $(n=2)$ and aggressive-invasive $(n=6)$. The annotation information of GeneChip was available based on the GPL2895 microarray platform (GE Healthcare/Amersham Biosciences CodeLink Human Whole Genome Bioarray).

The original probe-level data in the CEL files were converted into expression values with a $\log _{2}$ transformation. The probe signal was subsequently converted into the corresponding gene symbol using the microarray platform, GPL2895. For genes that corresponded with multiple probe sets, which exhibited a plurality of expression values, the expression values of those probe sets were averaged and the boxplot of the standardization expression value was mapped. Finally, these data were normalized using the between array normalization function in the limma package of $\mathrm{R}$ language (part of the Bioconductor project) $(16,17)$.

DEGs analysis and screening. The limma package in $\mathrm{R}$ language was used to identify DEGs in the invasive and aggressive-invasive samples by comparison with the non-invasive samples. The DEGs with a fold change value $\left(\log _{2} \mathrm{FCl}\right)>1$ and $\mathrm{P}<0.05$ were selected.

To avoid the difference of expression value in one group being greater than that between groups, the standard deviation (SD) of expression values of DEGs in the different groups was calculated. $\mathrm{SD}<0.15$ served as the cut-off criterion for further DEG screening.

The expression values of DEGs were hierarchically clustered by Cluster software (http://bonsai.hgc.jp/ mde hoon/software/cluster/software.htm) (18) and the differences in gene expression between non-invasive, invasive, and aggressive-invasive samples were intuitively observed. The expression values of overlapping genes between invasive and aggressive-invasive samples were also hierarchically clustered.

Functional enrichment analysis of DEGs. The functional enrichment analysis for the screened DEGs from the invasive and aggressive-invasive samples was performed using DAVID (19) for Kyoto Encyclopedia of Genes and Genomes (KEGG) (20) pathway and GO (21) function analysis. $\mathrm{P}<0.01$ served as the cut-off criterion. Comparison of the significant
GO terms and KEGG pathways were used to identify the different biological functions between the invasive and aggressive-invasive samples.

Co-expression network analysis. The correlation coefficient ( $R$ value) of DEGs between non-invasive and aggressive-invasive samples was calculated via the Spearman method of cor( ) function in R language (22). The invasive samples were excluded due to the low number of samples. The linear association of the expression value between two genes was verified using the cor( ) test and only the gene pairs with $|\mathrm{R}|>0.95$ and $\mathrm{P}<0.05$ were selected to construct the interaction networks.

\section{Results}

Screening differentially expressed genes. The publicly available microarray dataset, GSE22812 was preprocessed and a total of 7,579 genes were obtained from 13 PRL tumor samples. The differences between samples were significantly reduced following normalization using the between array normalization function (Fig. 1). The median of the gene expression value was almost on a straight line, indicating a marked degree of standardization.

At $\mid \log _{2} \mathrm{FCl}>1, \mathrm{P}<0.05$ and $\mathrm{SD}<0.15$, a total of 61 DEGs were obtained by comparing non-invasive and invasive samples, including 31 upregulated and 30 downregulated genes (Table I). A total of 89 DEGs were identified between non-invasive and aggressive-invasive samples, including 36 upregulated and 53 downregulated genes (Table II). The number of abnormally expressed genes from the aggressive-invasive samples was greater compared with the invasive samples.

Cluster analysis. Cluster analysis was used to determine the expression value of DEGs from the different samples. One aggressive-invasive sample (PRL tumor_agressive-invasive_6) showed a different expression value and was excluded. The other five aggressive-invasive, two invasive and five non-invasive samples were clustered and the color contrast is shown in Fig. 2. Briefly, a cluster analysis of five aggressive-invasive and five non-invasive samples showed two clusters; upregulated and downregulated genes in the aggressive-invasive samples (Fig. 2A). Similarly, cluster analysis of two invasive and five non-invasive samples showed two clusters; upregulated and downregulated genes in the invasive samples (Fig. 2B).

There were four genes that were shared by the invasive and aggressive-invasive samples, including solute carrier family 2, facilitated glucose transporter member 11 (SLC2A11), importin 7 (IPO7), chromogranin B $(\mathrm{CHGB})$ and teneurin transmembrane protein $1(T E N M 1)$. Cluster analysis showed upregulation of TENMI and SLC2A11, and downregulation of IPO7 and $C H G B$ in the aggressive-invasive samples (Fig. 2C).

Gene function annotation. To determine the function of DEGs in a PRL tumor, the DEGs were mapped into the GO database. Table III shows the significant GO terms in which the DEGs were primarily located. In the invasive samples, the majority of DEGs were enriched in response to cyclic adenosine monophosphate and a glucocorticoid stimulus. By contrast, in the aggressive-invasive samples, the cell cycle was the most significant GO term and the other genes partially correlated 
Table I. Significant upregulated and downregulated differentially expressed genes (top 10 of each) from invasive samples.

\begin{tabular}{|c|c|c|}
\hline Gene & $\log _{2} \mathrm{FC}$ & P-value \\
\hline PDE4C & 1.40 & $9.60 \mathrm{E}^{-04}$ \\
\hline PHF19 & 2.01 & $1.00 \mathrm{E}^{-03}$ \\
\hline SLC2A11 & 1.34 & $1.40 \mathrm{E}^{-03}$ \\
\hline KRTAP19-1 & 1.23 & $1.50 \mathrm{E}^{-03}$ \\
\hline ANKRD33B & 2.09 & $1.60 \mathrm{E}^{-03}$ \\
\hline KLHL8 & 1.50 & $2.00 \mathrm{E}^{-03}$ \\
\hline GPR52 & 1.29 & $2.40 \mathrm{E}^{-03}$ \\
\hline EGFR-AS1 & 1.23 & $2.90 \mathrm{E}^{-03}$ \\
\hline PLCZ1 & 1.22 & $3.20 \mathrm{E}^{-03}$ \\
\hline ASGR1 & 1.06 & $4.40 \mathrm{E}^{-03}$ \\
\hline CDO1 & -2.33 & $4.89 \mathrm{E}^{-05}$ \\
\hline TAGLN3 & -1.46 & $4.65 \mathrm{E}^{-04}$ \\
\hline AKAP12 & -2.33 & $1.30 \mathrm{E}^{-03}$ \\
\hline CORO1C & -1.45 & $2.30 \mathrm{E}^{-03}$ \\
\hline LAPTM4B & -1.17 & $2.40 \mathrm{E}^{-03}$ \\
\hline JUNB & -1.46 & $3.30 \mathrm{E}^{-03}$ \\
\hline HPCAL1 & -1.63 & $4.00 \mathrm{E}^{-03}$ \\
\hline ACTN1 & -1.30 & $4.20 \mathrm{E}^{-03}$ \\
\hline HIST1H2AG & -1.45 & $5.70 \mathrm{E}^{-03}$ \\
\hline ATP1B3 & -1.08 & $6.20 \mathrm{E}^{-03}$ \\
\hline \multicolumn{3}{|c|}{ 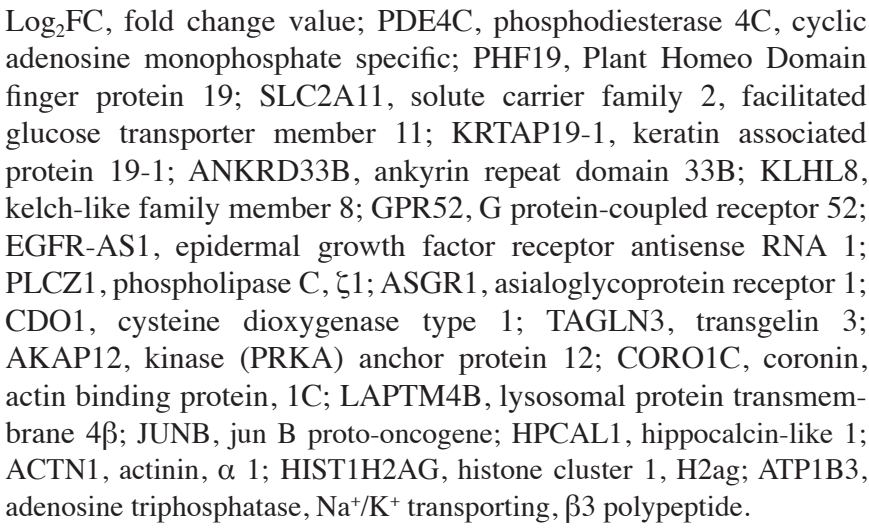 } \\
\hline
\end{tabular}

with the response to hormone stimulation (Table III). DEGs from the aggressive-invasive samples were enriched into GO terms compared with invasive samples. However, DEGs were not significantly enriched into a specific pathway via the KEGG pathway analysis, which may be due to the limitation of the low number of DEGs that were analyzed.

Construction of a co-expression network. To construct the co-expression network of DEGs between non-invasive and aggressive-invasive samples, the correlation coefficient of DEGs was calculated and only gene pairs with $|\mathrm{R}|>0.95$ and $\mathrm{P}<0.05$ were selected. The co-expression network of DEGs from the invasive samples consisted of 42 nodes and 34 co-expressed pairs, presenting scattered gene pairs and only one module with more than five nodes (Fig. 3A). In the aggressive-invasive samples, the co-expression network of DEGs contained 68 nodes and 122 co-expressed pairs gathered together, forming positive
Table II. Significant upregulated and downregulated differentially expressed genes (top 10 of each) from aggressive-invasive samples.

\begin{tabular}{lrl}
\hline Gene & Log $_{2} \mathrm{FC}$ & P-value \\
\hline CCNB1 & 1.71 & $1.29 \mathrm{E}^{-04}$ \\
RACGAP1 & 1.75 & $1.44 \mathrm{E}^{-04}$ \\
TAGLN & 1.97 & $2.01 \mathrm{E}^{-04}$ \\
ZNF430 & 1.38 & $3.14 \mathrm{E}^{-04}$ \\
GTDC1 & 1.62 & $3.67 \mathrm{E}^{-04}$ \\
SLC2A11 & 1.19 & $4.38 \mathrm{E}^{-04}$ \\
VLDLR & 1.19 & $5.50 \mathrm{E}^{-04}$ \\
DBF4 & 1.43 & $6.44 \mathrm{E}^{-04}$ \\
COL6A2 & 1.64 & $7.05 \mathrm{E}^{-04}$ \\
SRGAP2C & 1.23 & $8.93 \mathrm{E}^{-04}$ \\
RCN1 & -1.44 & $8.91 \mathrm{E}^{-04}$ \\
C11orf82 & -1.21 & $9.13 \mathrm{E}^{-04}$ \\
CLCN1 & -1.74 & $1.00 \mathrm{E}^{-03}$ \\
IPO7 & -1.38 & $1.50 \mathrm{E}^{-03}$ \\
SLC23A1 & -1.22 & $1.50 \mathrm{E}^{-03}$ \\
SIK2 & -1.43 & $1.98 \mathrm{E}^{-03}$ \\
STAT5B & -1.32 & $2.00 \mathrm{E}^{-03}$ \\
ZNF516 & -1.28 & $2.10 \mathrm{E}^{-03}$ \\
GLI1 & -1.51 & $2.50 \mathrm{E}^{-03}$ \\
CCHCR1 & -1.21 & $2.60 \mathrm{E}^{-03}$ \\
\hline
\end{tabular}

$\log _{2} \mathrm{FC}$, fold change value; CCNB1, cyclin B1; RACGAP1, Rac guanosine triphosphatase activating protein 1; TAGLN, transgelin; ZNF430, zinc finger protein 430; GTDC1, glycosyltransferase-like domain containing 1; SLC2A11, solute carrier family 2 (facilitated glucose transporter), member 11; VLDLR, very low density lipoprotein receptor; DBF4; DBF4 homolog (S. cerevisiae); COL6A2, collagen, type VI, $\alpha 2$; SRGAP2C, SLIT-ROBO @ guanosine triphosphatase activating protein $2 \mathrm{C}$; RCN1, reticulocalbin 1 , EF-hand calcium binding domain; C11orf82, chromosome 11 open reading frame 82; CLCN1, chloride channel, voltage-sensitive 1; IPO7, importin 7; SLC23A1, solute carrier family 23 (ascorbic acid transporter), member 1; SIK2, salt-inducible kinase 2; STAT5B, signal transducer and activator of transcription 5B; ZNF516, zinc finger protein 516; GLI1, glioblastoma family zinc finger 1; CCHCR1, coiled-coil $\alpha$-helical rod protein 1 .

correlation modules and mixed modules (Fig. 3B). There were five modules containing more than five nodes (Fig. 3B).

Briefly, SLC2A11 and $C H G B$ were shared by two co-expression networks within the different co-expressed pairs. Furthermore, no co-expressed pair simultaneously occurred in two co-expression networks. A number of downregulated DEGs in the aggressive-invasive samples were gathered to form positive correlation modules (Fig. 3B).

\section{Discussion}

PRL-secreting adenomas were the most common ( 40\%) type of pituitary adenomas and an important cause of hypogonadism, infertility and osteoporosis, as well as central compressive effects $(8,23,24)$. Medical therapy with dopamine agonists are highly effective in controlling the tumor mass in the majority 

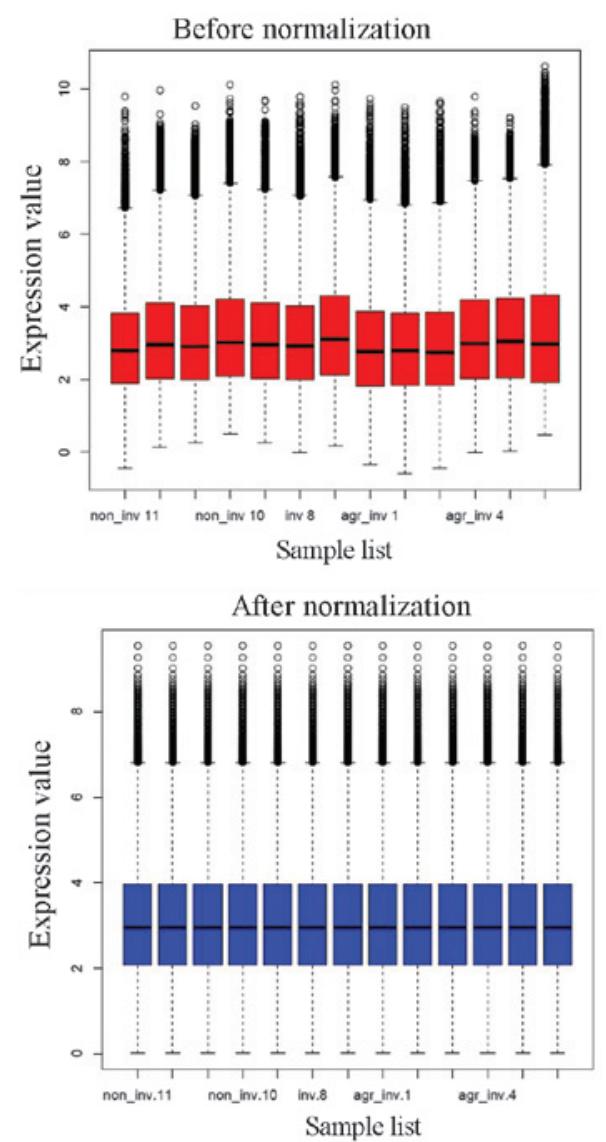

Figure 1. Boxplot of gene expression values before (top) and after (bottom) normalization. Abscissa axis, samples; vertical axis, gene expression value; black horizontal line, median of the gene expression value for each sample.

of cases, however, complicated situations, including dopamine agonist resistance, pregnancy and malignant prolactinomas, may require multi-therapies involving surgery, radiotherapy, or a combination of the two $(25,26)$. Progress in elucidating the natural development of prolactinomas was beneficial for disease management (27). However, currently there is no method to accurately identify the invasive pituitary tumors that are most likely to metastasize, which would enable early treatment prior to progression to malignant prolactinomas.

In the current study, a total of 61 and 89 DEGs were identified by comparing non-invasive samples with invasive and aggressive-invasive samples, respectively. Cluster analysis showed that four overlapping genes, SLC2A11, TENM1, IPO7 and $C H G B$ were co-expressed, indicating the close association of these genes with tumor transformation. It has been reported that $C H G B(C g B)$ may be considered as a universal granular marker for pituitary adenomas (28). In addition, the hypothalamic hormone, gonadotropin-releasing hormone and the CgA-derived peptide, pancreastatin, may regulate $C g B$ mRNA in gonadotroph adenomas, indicating an autocrine effect of pancreastatin on pituitary tumor function (29). $C g B$ is a precursor of two peptides, GAWK (CHGB 420-493) and BAM-1745 (1745-dalton pyroglutamyl) (30). Although the other three genes have not been verified to be associated with pituitary adenomas, previous studies have implicated these genes in other cancers. Notably, $\beta$-karyopherin genes (IPO7) were the importin- $\alpha / \beta$ complex that was frequently overexpressed in cancer and is
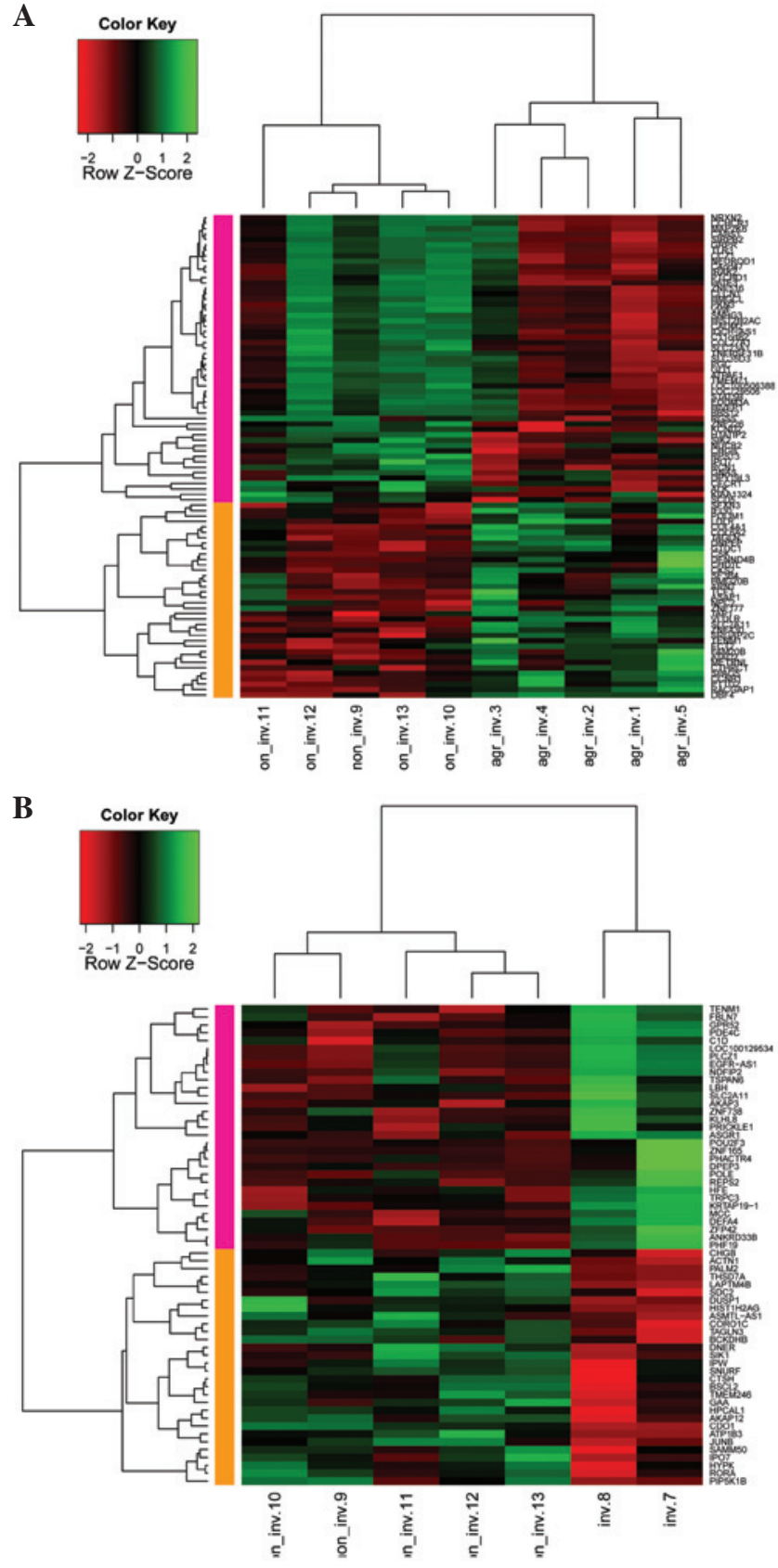

C

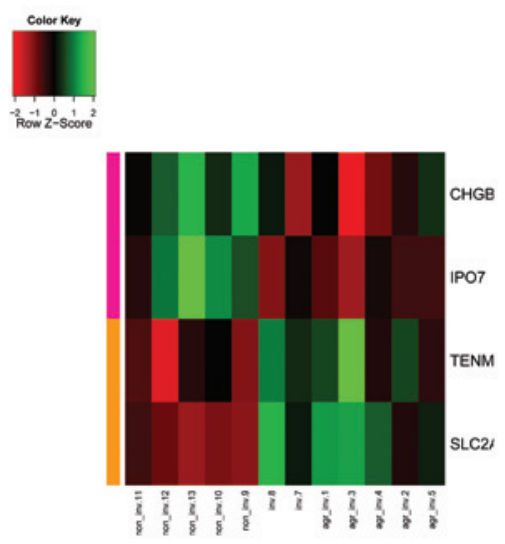

Figure 2. Cluster analysis of differentially expressed genes (DEGs) from five non-invasive, two invasive and five aggressive-invasive samples. Cluster analysis of DEGs from; (A) five non-invasive and five aggressive-invasive samples and (B) five non-invasive and two invasive samples. (C) Cluster analysis of four overlapping DEGs. Lower abscissa axis, different samples; upper abscissa axis, clusters of samples; left vertical axis, clusters of DEGs; right vertical axis, DEGs; red, downregulation; and green, upregulation. 
Table III. Significantly enriched GO terms in prolactin tumor cells from invasive and aggressive-invasive samples with $\mathrm{P}<0.01$.

\begin{tabular}{|c|c|c|c|c|}
\hline Sample & Category & GO Term & Count & P-value \\
\hline \multirow[t]{3}{*}{ Invasive } & GOTERM_BP_FAT & $\begin{array}{l}\text { GO:0051591 response to cyclic adenosine } \\
\text { monophosphate }\end{array}$ & 4 & 0.0003 \\
\hline & GOTERM_BP_FAT & GO:0051384 response to glucocorticoid stimulus & 4 & 0.0018 \\
\hline & GOTERM_BP_FAT & GO:0031960 response to corticosteroid stimulus & 4 & 0.0023 \\
\hline \multirow[t]{7}{*}{ Aggressive-invasive } & GOTERM_BP_FAT & GO:0045637 regulation of myeloid cell differentiation & 4 & 0.0042 \\
\hline & GOTERM_BP_FAT & GO:0022402 cell cycle process & 9 & 0.0045 \\
\hline & GOTERM_BP_FAT & GO:0010033 response to organic substance & 10 & 0.0059 \\
\hline & GOTERM_BP_FAT & GO:0007049 cell cycle & 10 & 0.0094 \\
\hline & GOTERM_CC_FAT & GO:0005576 extracellular region & 19 & 0.0032 \\
\hline & GOTERM_CC_FAT & GO:0044421 extracellular region part & 12 & 0.0040 \\
\hline & GOTERM_MF_FAT & GO:0046982 protein heterodimerization activity & 6 & 0.0029 \\
\hline
\end{tabular}

GO, Gene Ontology.

A

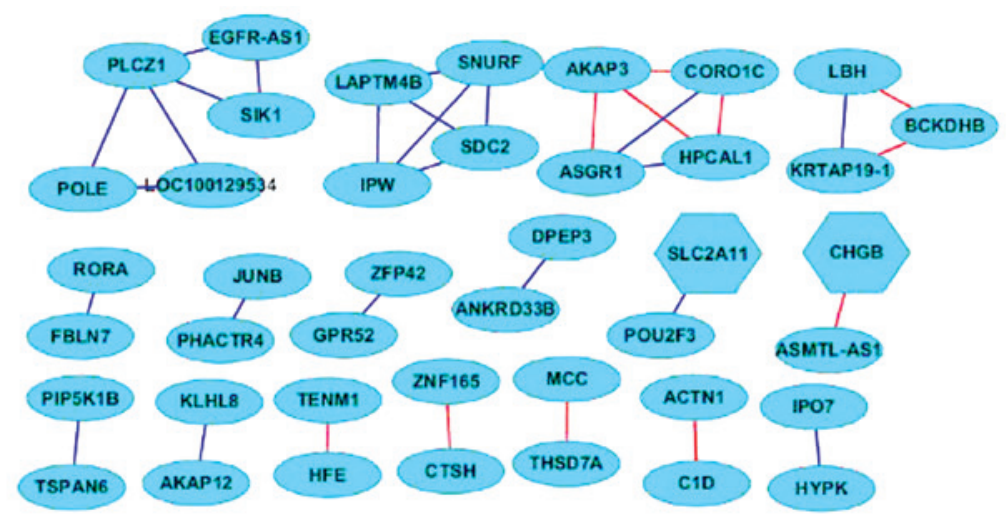

$\mathbf{B}$

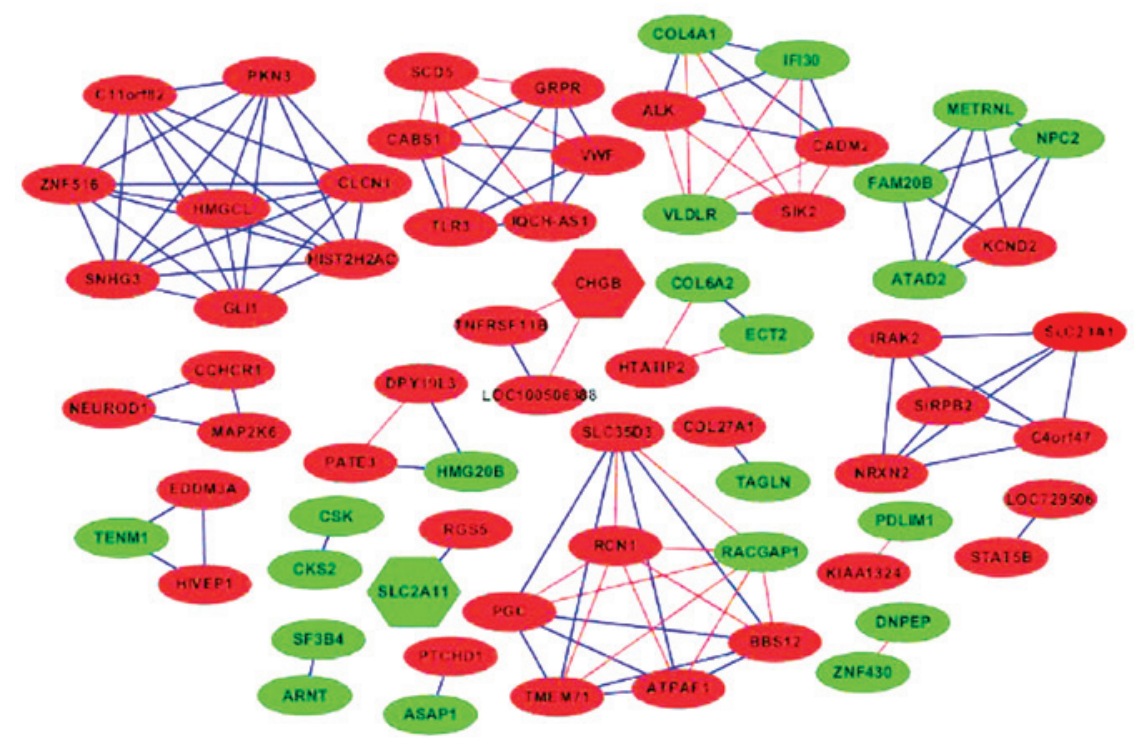

Figure 3. Co-expression networks of differentially expressed genes (DEGs) in (A) non-invasive and (B) aggressive-invasive samples. Blue line, significant positive correlation of gene pairs in a prolactin pituitary tumor; red line, significant negative correlation of gene pairs. (A) The blue nodes presented DEGs from non-invasive and aggressive-invasive samples. (B) Red nodes, downregulated DEGs in aggressive-invasive samples compared with non-invasive samples; green nodes, upregulated DEGs; and hexagonal nodes (A and B), CHGB and SLC23A11 genes.

mediated by the nuclear importing of proteins with a classical nuclear localization signal $(31,32)$. SLC $2 A 11$, the solute carrier 2A11 gene, encodes a novel sugar transporter, Human glucose transporter 11, and consists of 12 exons, located on chromosome 22q11.2. In human tissue, a 7.2-kb transcript of SLC2A11 was detected exclusively in heart and skeletal muscle (33). In verte- 
brates, there are four paralogs, TENM 1-4, which are expressed prominently in the developing central nervous system. TENMI and -2 are expressed by distinct interconnected populations of neurons (34). Based on their distinct complementary expression, a possible function in the establishment of proper connectivity in the brain was hypothesized (35). Progress in elucidating the mechanisms underlying the pathogenesis of prolactinomas may enable future development of novel molecular therapies for treatment-resistant cases.

$\mathrm{GO}$ function enrichment analysis showed different GO terms of invasive and aggressive-invasive samples, indicating a different biological progression in the two stages. Hormone genes were abnormally expressed in the invasive samples and, in the aggressive-invasive samples, dysregulation of cell cycle progression was hypothesized as significant in malignant metastasis. In addition, the co-expression network of DEGs showed that $S L C 2 A 11$ and $C H G B$ occurred in two co-expression networks combined with different co-expressed pairs. These results indicated that $C H G B$ and $S L C 2 A 11$ may be significant in PRL tumor progression and may serve as molecular biomarkers for PRL tumors. However, samples were limited in the current study and co-expression of metastasis-associated genes was not comprehensively elucidated.

In conclusion, four genes that are relevant to tumor metastasis were identified from 13 PRL tumor samples using a bioinformatics method. Two gene pairs were shared by co-expression networks of DEGs from invasive and aggressive-invasive samples. These candidate factors correlated with the progression of prolactinoma and, thus, may provide a series of potential therapeutic targets for the treatment of pituitary tumors.

\section{Acknowledgements}

The present study was supported by a grant from the Natural Science Foundation Project of CQ CSTC (grant no. 2010BB5179).

\section{References}

1. Mindermann T and Wilson CB: Age-related and gender-related occurrence of pituitary adenomas. Clin Endocrinol (Oxf) 41: 359-364, 1994

2. Dessimoz C,Browaeys P, MaederP, et al: Transformation of a microprolactinoma into a mixed growth hormone and prolactin-secreting pituitary adenoma. Front Neuroendocrin (Lausanne) 2: 116, 2011.

3. Daly AF, Rixhon M, Adam C, Dempegioti A, Tichomirowa MA and Beckers A: High prevalence of pituitary adenomas: a cross-sectional study in the province of Liege, Belgium. J Clin Endocrinol Metab 91: 4769-4775, 2006.

4. Ma W, Ikeda H and Yoshimoto T: Clinicopathologic study of 123 cases of prolactin-secreting pituitary adenomas with special reference to multihormone production and clonality of the adenomas. Cancer 95: 258-266, 2002.

5. Ahtiainen P, Sharp V, Rulli SB, et al: Enhanced LH action in transgenic female mice expressing hCGbeta-subunit induces pituitary prolactinomas; the role of high progesterone levels. Endocr Relat Cancer 17: 611-621, 2010.

6. Ali S, Miller KK and Freudenreich O: Management of psychosis associated with a prolactinoma: case report and review of the literature. Psychosomatics 51: 370-376, 2010.

7. Gillam MP, Molitch ME, Lombardi G and Colao A: Advances in the treatment of prolactinomas. Endocr Rev 27: 485-534, 2006.

8. Vlotides G, Cooper O, Chen YH, Ren SG, Greenman Y and Melmed S: Heregulin regulates prolactinoma gene expression. Cancer Res 69: 4209-4216, 2009.

9. Leriche VK, Asa SL and Ezzat S: Epidermal growth factor and its receptor (EGF-R) in human pituitary adenomas: EGF-R correlates with tumor aggressiveness. J Clin Endocrinol Metab 81: 656-662, 1996.
10. Roncaroli F, Nosé V, Scheithauer BW, et al: Gonadotropic pituitary carcinoma: HER-2/neu expression and gene amplification. Report of two cases. J Neurosurg 99: 402-408, 2003.

11. Onguru O, Scheithauer BW, Kovacs K, et al: Analysis of epidermal growth factor receptor and activated epidermal growth factor receptor expression in pituitary adenomas and carcinomas. Mod Pathol 17: 772-780, 2004.

12. Zhang H, Berezov A, Wang Q, et al: ErbB receptors: from oncogenes to targeted cancer therapies. J Clin Invest 117: 2051-2058, 2007.

13. Tzahar E, Waterman H, Chen X, et al: A hierarchical network of interreceptor interactions determines signal transduction by Neu differentiation factor/neuregulin and epidermal growth factor. Mol Cell Biol 16: 5276-5287, 1996.

14. Vlotides G, Siegel E, Donangelo I, Gutman S, Ren SG and Melmed S: Rat prolactinoma cell growth regulation by epidermal growth factor receptor ligands. Cancer Res 68: 6377-6386, 2008.

15. Wierinckx A, Roche M,Raverot G, et al: Integrated genomic profiling identifies loss of chromosome 11p impacting transcriptomic activity in aggressive pituitary PRL tumors. Brain Pathol 21: 533-543, 2011.

16. Toedling J, Sklyar O, Huber W, et al: Ringo - an R/Bioconductor package for analyzing ChIP-chip readouts. BMC Bioinformatics 8: 221, 2007.

17. Bolstad BM, Irizarry RA, Astrand M and Speed TP: A comparison of normalization methods for high density oligonucleotide array data based on variance and bias. Bioinformatics 19: 185-193, 2003.

18. Eisen MB, Spellman PT, Brown PO and Botstein D: Cluster analysis and display of genome-wide expression patterns. Proc Natl Acad Sci USA 95: 14863-14868, 1998.

19. Huang da W, Sherman BT and Lempicki RA: Systematic and integrative analysis of large gene lists using DAVID bioinformatics resources. Nat Protoc 4: 44-57, 2009.

20. Kanehisa M: The KEGG database. Novartis Found Symp 247: 91-103; discussion 119-128, 244-152, 2002.

21. Hulsegge I, Kommadath A and Smits MA: Globaltest and GOEAST: two different approaches for Gene Ontology analysis. BMC Proc 3 (Suppl 4): S10, 2009.

22. Becker RA, Chambers JM and Wilks AR. The New S Language: A Programming Environment for Data Analysis and Graphics. Vol 1. Chapman \& Hall, London, 1988.

23. Melmed S: Mechanisms for pituitary tumorigenesis: the plastic pituitary. J Clin Invest 112: 1603-1618, 2003.

24. Casanueva FF, Molitch ME, Schlechte JA, et al: Guidelines of the Pituitary Society for the diagnosis and management of prolactinomas. Clin Endocrinol (Oxf) 65: 265-273, 2006.

25. Olafsdottir A and Schlechte J: Management of resistant prolactinomas. Nat Clin Pract Endocrinol Metab 2: 552-561, 2006.

26. Whitelaw BC, Dworakowska D, Thomas NW, et al: Temozolomide in the management of dopamine agonist-resistant prolactinomas. Clin Endocrinol (Oxf) 76: 877-886, 2012.

27. Ragel BT and Couldwell WT: Pituitary carcinoma: a review of the literature. Neurosurg Focus 16: E7, 2004.

28. Riva C, Leutner M, Capella C, et al: Different expression of chromogranin A and chromogranin B in various types of pituitary adenomas. Zentralbl Pathol 139: 165-170, 1993.

29. Jin L, Scheithauer BW, Young WF Jr, Davis DH, Klee GG and Lloyd RV: Pancreastatin secretion by pituitary adenomas and regulation of chromogranin B mRNA expression. Am J Pathol 148: 2057-2066, 1996

30. Louthan O: Chromogranin a in physiology and oncology. Folia Biol (Praha) 57: 173-181, 2011.

31. Golomb L, Bublik DR, Wilder S, et al: Importin 7 and exportin 1 link c-Myc and p53 to regulation of ribosomal biogenesis. Mol Cell 45: 222-232, 2012.

32. Atasheva S, Fish A, Fornerod M and Frolova EI: Venezuelan equine Encephalitis virus capsid protein forms a tetrameric complex with CRM1 and importin alpha/beta that obstructs nuclear pore complex function. J Virol 84: 4158-4171, 2010.

33. Doege H, Bocianski A, Scheepers A, et al: Characterization of human glucose transporter (GLUT) 11 (encoded by SLC2A11), a novel sugar-transport facilitator specifically expressed in heart and skeletal muscle. Biochem J 359: 443-449, 2001.

34. Kenzelmann D, Chiquet-Ehrismann R, Leachman NT and Tucker RP: Teneurin-1 is expressed in interconnected regions of the developing brain and is processed in vivo. BMC Dev Biol 8: $30,2008$.

35. Beckmann J, Vitobello A, Ferralli J, Kenzelmann Brož DK, Rijli FM and Chiquet-Ehrismann R: Human teneurin-1 is a direct target of the homeobox transcription factor EMX2 at a novel alternate promoter. BMC Dev Biol 11: 35, 2011. 